\title{
A Review on Emotional Evaluations for Smart Phone
}

\author{
Amaka Mercy Udengwu, Tek Yong Lim, and Soon-Fatt Cheong \\ Faculty of Computing and Informatics, Multimedia University, Jalan Multimedia, \\ 63100 Cyberjaya, Selangor, Malaysia \\ lyamy2001@yahoo.com, \{tylim,sfcheong\}@mmu.edu.my
}

\begin{abstract}
Human Computer Interaction (HCI) has become progressively more concerned with user experience and emotions. A variety of what experience and emotions is has been expressed in modern years which propose an amount of vital insights but it is unclear on how they inform design. The aim of this paper is to gain a deeper understanding on the techniques to collect and evaluate emotional responses conducted by other researchers. Four evaluation methods were reviewed, namely valence method, self-reported method, experiment method and semantic differential method.
\end{abstract}

Keywords: Emotional Design, Smart Phone, Evaluation.

\section{$1 \quad$ Introduction}

Recently, emotion is playing an important role in customer interaction with the products [1].The evaluation of customers experiences help to determines various perspectives. In this regard, Kansei engineering proposed by [2, 3] is one of the consistent and practical methodologies in dealings with customer's emotional needs.

Emotional situations are frequently incorporated in the explanation of customer requirements and evaluations. Products that reflect on emotion responses and inducing positive emotions tend to draw customers. Thus, emotional design model has been proposed to integrate customer's emotional requirements into design elements to convey customer emotional satisfaction [4].

Emotional design model proposed that design elements can be associated to three different levels of emotional responses, namely visceral, behavioral and reflective. Visceral responses are concerned with aesthetic measures. Behavioral responses are related to the usability and effectiveness of use. Reflective responses are simulated by the rationalization and intellectualization of a product. Besides that, product also can evoke a wide range of emotions, both negative and positive. Negative emotions stimulate individuals to reject or withdraw from the object while a positive emotion stimulates individuals to accept the object.

\section{$2 \quad$ Literature Review}

This paper reviews different methods used by other researchers to elicit and analyze emotional responses from users while interacting with an interactive product such as smart phone. 


\subsection{Valence Method}

Valence method was developed to gather customer experience during evaluations. It was able to elicit every possible detail, extract aspect of product that causes good and bad customer experience [5]. It is significant for designers to recognize which design aspects have caused a good (positive) or bad (negative) experience. This method involved two phases, namely exploration phase and retrospective interview phase.

In the exploration phase, users explored product in whichever way that they like best. There was no pre-defined task given to users. Whenever customers experienced positive and negative emotions, their feelings were spotted by pressing colored button on remote control (either a green button for positive feelings or red button for negative feelings). The button presses were recorded as valence markers with time stamps in a video recording.

In the retrospective interview phase, two main aspects were investigated: (1) product attribute design that caused setting of the valence marker and (2) the underlying customer needs. Each valence marker is discussed in order to recognize the design aspect of a product causing the experience, its meaning to customer and customer's underlying needs. The outcome of this phase was a verbal protocol for each marker with the following properties: attribute design, meaning for the customer and underlying customer needs.

\section{$2.2 \quad$ Self-reported Method}

The self-reported method adopted both verbal and non-verbal scales for gathering information about user's emotions [6]. The advantage of this method is that researchers do not need to be present. Three variations of self-reported method were identified, namely experience clip, expressing emotions and experiences (3E), and feedback app.

Experience clip was introduced by [7]. In this method, two friends would work together to evaluate a product where one person would capture a short video clip of his/her friend using the product. Since the video clip was taken by a friend, user can express his/her emotion easier. This method also enabled them to share and compare experiences with each other after they switched roles during the evaluation.

$3 \mathrm{E}$ was a controlled way of expressing user emotions by scribbling in a diary. It can be a drawing, written expression or combination of both. However, it can be very challenging for researchers to analyze these outputs because each symbolizes user inner feelings. Thus, each user was interviewed in order to understand the diary content. In other words, this semi-structured interview allowed researchers to get deeper information of what users have drawn and written in diary.

Feedback app was a mobile application used to capture user emotions at different times, especially straight away after an interaction being completed. This is also known as experience sampling method (ESM). In this app, users would be prompted using a predefined question and choose one of the nine emoticons as their emotions. This method also integrated both application to be tested and emotion gathering instrument on the same device. 


\subsection{Experiment Method}

Attractiveness of a product appearance can influence perceive usability and [8] carried out an experiment method to investigate the effect of attractiveness, effectiveness, and efficiency on perceived usability. Participants were asked to use mobile phones to perform nine different tasks such as place a call, change assigned ringer and others. Then, they rated each mobile phone with respect to usability and attractiveness. The result revealed that participants who can use a phone effectively and efficiently to complete common phone tasks, tend to rate the phone high in usability whereas participant who cannot, tend to rate the phone low in usability. In short, attractive products were rated high in usability than unattractive products.

\subsection{Semantic Differential Method}

User's first choice is one of the vital user requirements. Therefore, it becomes a difficult job for product designers to convey user's unspoken first choice into detailed design specification [9]. The semantic differential method examined the association between user's first choice impression of mobile phones and design element. The semantic differential method is comprised of two steps, namely (1) build a measurement scale for preference study, and (2) measure the image perception and user's preference.

The goal of first step was to derive a set of image words to be used as measurement scale. Thus, experienced designers were selected to evaluate forty mobile devices and eleven pairs of image words were obtained. The second step was to determine the relationship between user preference and product design element. 102 participants were asked to assess 26 mobile devices using the eleven pairs of image words. The result showed that participants preferred soft and compact mobile phone.

\section{Discussion and Conclusion}

Self-reported method, valence method, experiment method and semantic differential method are various ways of eliciting user emotion in different circumstances. Our future work will use web-based questionnaire to elicit emotional response from participants in three different levels of emotional design model [1]. Visceral level will be conducted through exploration, whereby participants are expected to see and feel the smart phones, and required to enter their first impression. Behavioral level will be measured through perceived usability of the smart phones. Participants are also required to enter their emotional response after performing some tasks such as place a call and send a text message. In reflective level, participants are expected to make purchase decision by using laddering techniques.

Acknowledgement. This work is supported by Malaysian Ministry of Higher Education (MOHE) under the Fundamental Research Grant Scheme (FRGS/2/2010/SG/MMU/03/4). 


\section{References}

1. Norman, D.A.: Preface. In: Schifferstein, H.N.J., Hekkert, P. (eds.) Product Experience, pp. xix-xx. Elsevier, San Diego (2008)

2. Nagamachi, M.: Perspectives and the New Trend of Kansei/Affective Engineering. TQM Journal 20(4), 290-298 (2008)

3. Nagamachi, M.: Kansei Engineering: A New Ergonomic Consumer-Oriented Technology For Product Development. International Journal of Industrial Ergonomics 15(1), 3-11 (1995)

4. Norman, D.A.: Emotional Design: Why Do We Love (Or Hate) Everyday Things. Basic Books, New York (2004)

5. Burmester, M., Mast, M., Jager, K., Homans, H.: Valence Method for formative evaluation of user experience. In: Proceedings of the 8th ACM Conference on Designing Interactive Systems, Stuttgart, Germany, pp. 364-367 (2010)

6. Isomursu, M., Tahti, M., Vainamo, S., Kuutti, K.: Experimental evaluation of five methods for collecting emotions in field settings with mobile applications. International Journal of Human-Computer Studies 65(4), 404-418 (2009)

7. Isomursu, M., Kuutti, K., Väinämö, S.: Experience Clip: Method For User Participation and Evaluation of Mobile Concepts. In: Proceedings of the Participatory Design Conference, pp. 83-92 (2004)

8. Quinn, J.M., Tran, T.Q.: Attractive phones don't have to work better: independent effects of attractiveness, effectiveness and efficiency on perceived usability. In: Proceedings of the SIGCHI Conference on Human Factors in Computing Systems, Atlanta, GA, USA, pp. 353-362 (2010)

9. Chuang, M.C., Chang, C.C., Hsu, S.H.: Perceptual factors underlying user preferences towards product form of mobile phones. International Journal of Industrial Ergonomics 27(4), 247-258 (2001) 